\title{
Handwritten Courtesy Amount and Signature Recognition on Bank Cheque using Neural Network
}

\author{
Mohammad Badrul Alam \\ Miah \\ Dept. of Information and \\ Communication Technology \\ Mawlana Bhashani Science \\ and Technology University, \\ Bangladesh.
}

\author{
Mohammad Abu Yousuf, \\ Ph.D \\ Institute of Information \\ Technology (IIT), \\ Jahangirnagar University, \\ Bangladesh.
}

\author{
Md. Sohag Mia \\ Dept. of Information and \\ Communication Technology \\ Mawlana Bhashani Science \\ and Technology University, \\ Bangladesh.
}

\author{
Md. Parag Miya \\ Dept. of Information and Communication Technology \\ Mawlana Bhashani Science and Technology University, Bangladesh.
}

\begin{abstract}
Bank cheques are used not only in our country but also all over the world for financial transactions. Still now Bank cheques are processed manually everyday in both developed and developing countries. The proposed system presents a recognition system of both handwritten courtesy amount and signature .To read the courtesy amount and signature, the system uses the scanned image of Bank cheque. The proposed system divided into several stages that focus on: Image preprocessing; the detection of block of courtesy amount and signature; the post processing; the segmentation of string into characters; Feature extraction of courtesy amount and signature; Neural Network recognition. At first, scanned image is converted into gray image and then it is filtered. Then detection of the courtesy amount and signature is performed using image cropping method. The cropped images are post-processed to ensure correct recognition. Then courtesy amount is segmented using the segmentation method. The segmentation module has been implemented as a recursive process. Then segmented digit and binary image of signature are passed through the feature extraction process. A rotation, translation, scaling and orientation invariant feature extraction method has been used to extract the features of the input images based on moment feature extraction method. And finally, a back propagation learning algorithm is used to train up the network and tested the performance. Overall success rate of the system is tested in different sorts of numeral and the experimental result shows satisfactory performance.
\end{abstract}

\section{Keywords}

Bank Check, Courtesy Amount, Signature, Segmentation, Neural Network Recognition.

\section{INTRODUCTION}

Now a day, automatic bank check processing is an interesting field in banking industry. As a large number of bank checks are still processed manually that involves the manual reading of the checks and inputting their respective values into the computer, this manual procedure involves high cost due to its labor-consuming operation. Handwritten text and signature in the bank check is an important obstacle towards the automation of the bank check processing. So the proposed system has been design to recognize handwritten text and signature from bank check.

In Bangladesh, author's information on bank check is frequently handwritten. Extraction of this information involves detection, localization, tracking, enhancement, and recognition of the text from a given image of bank check. A system that is able to read check automatically would be very helpful, especially if it is fast and accurate. Even if misclassification occurs, the mistake could be detected potentially during the recognition process; however it is more desirable that the system rejects a check in case of doubt so that it can be directed to manual processing from the beginning. All these factors motivated us to carry out an experiment for automatic recognition of handwritten courtesy amount and signature on bank checks.

The recognition of machine-printed documents has been a very successful application. In contrast, it is more difficult for computer systems to read handwritten texts and numbers. In the processing of handwritten fields, computer systems are generally slower and yield less accurate results than humans [2], [5]. Segmentation of the courtesy amount into individual digits is the most critical task in check processing [18]. This includes the separation of touching characters and the merging of character fragments with other pieces. The segmentation of connected numbers is the main bottleneck in the handwritten numeral recognition system [17]. There are studies in the literature on locating the courtesy amount and signature block on bank checks [7-9], [11], [16], and [20]. Several researches have been carried out in the field of character recognition system [12], [14]. However, there are no precise methods to recognize handwritten courtesy amount and signature simultaneously from bank check. For this reason, we attempt to make a system for bank management to make their daily management flexible and faster through automated recognition of handwritten courtesy amount and signature on bank checks.

The structure of the paper is approximately as follows: Section-2 describes the problems or methods to be solved. In Section-3 discusses about the proposed system. In section-4 discusses the Implementation, Results and Performance of the proposed system. And finally discuss the conclusion in section-5.

\section{THE RESEARCH METHOD}

Bank check is consisted of many fields which may be handwritten or printed. Courtesy amount block contain the total amount that is written in numbers. The bank check also contains the name of the recipient, the date, the amount to be paid (textual format), the courtesy amount (numerical format) and the signature of the person who wrote the check as well as symbols and graphics [18]. The official value of the check is the amount written in words which is often called as the "legal 
amount". However, the courtesy amount is more effective in the automation of bank check processing. There are many types of bank check used in Bangladesh one of them are shown in Figure 1.

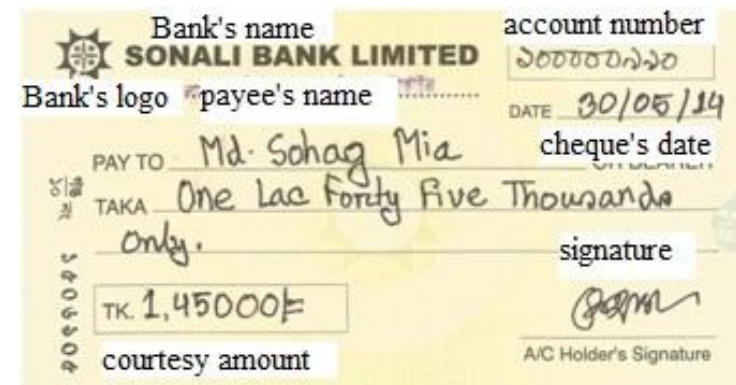

Fig 1: General format of Bank checks used in Bangladesh.

\section{THE PROPOSED SYSTEM}

The proposed system is designed for automatic detection and recognition of courtesy amount and signature from bank check. The system consists of five modules: (i) Image Preprocessing (ii) Extraction of courtesy amount and signature (iii) Binarization of courtesy amount and signature (iv) Segmentation of digits (v) Recognition using artificial neural network. Figure 2 shows the overall system architecture.

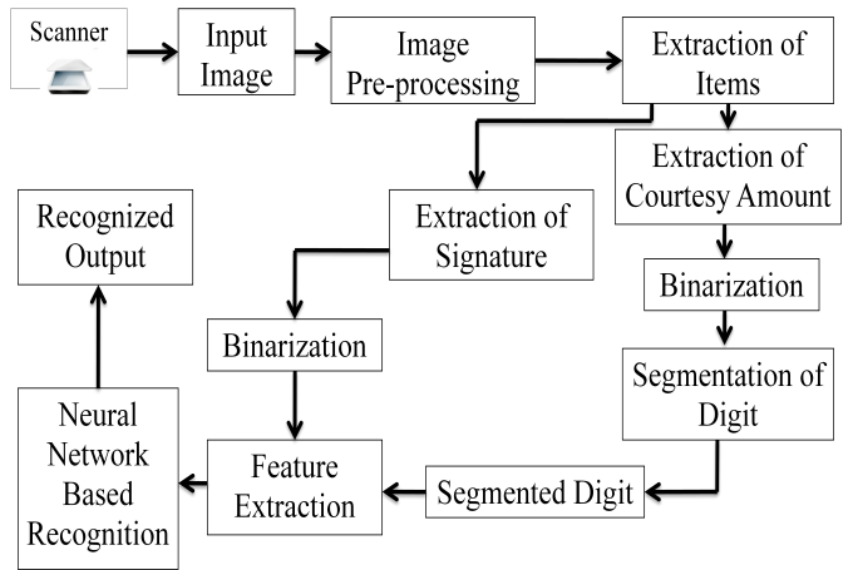

Fig 2: Block diagram of the proposed system.

\subsection{Image acquisition}

Image acquisition is the process of obtaining a digitized image from a real world source. Today, acquisition is usually done using different kinds of input devices such as scanner, digital camera, web camera, PDA, camcorder etc[15]. Here we proposed to use scanner to acquire the image of the bank check. The scanned image is shown in Figure 3.

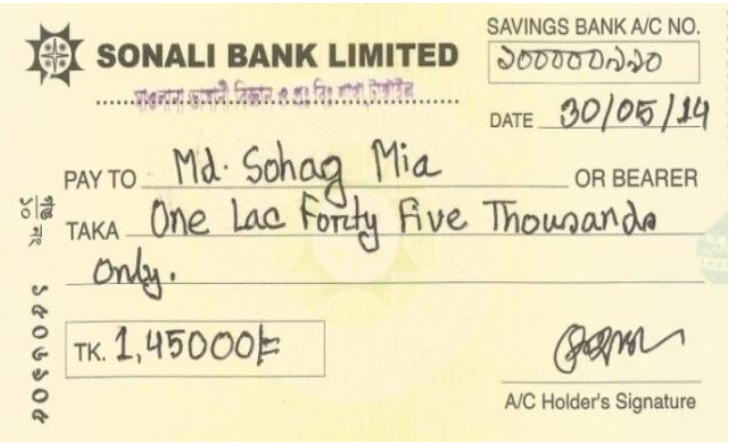

Fig 3: Scanned image of Bank Check.

\subsection{Image Pre-processing}

Images that are acquired from documents containing text and numerical string, passed through the preprocessing steps [20]. Figure 4 shows the block diagram of image pre-processing steps. This sub section describes each steps of image pre processing.

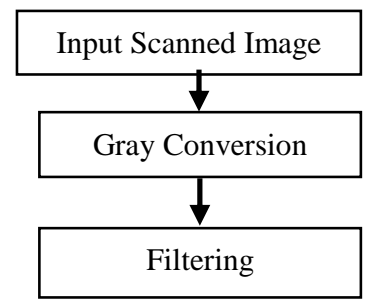

Fig 4: Block diagram of image preprocessing steps

\subsubsection{Gray Scale Conversion}

RGB image is converted into gray scale image using NTSC gray scale conversion which is frequently used to convert RGB to gray scale conversion. The equation is given bellow:

Grayscale value $=0.3 \times$ Red $+0.59 \times$ Green $+0.11 \times$ Blue Grayscale image is shown in Figure 5.

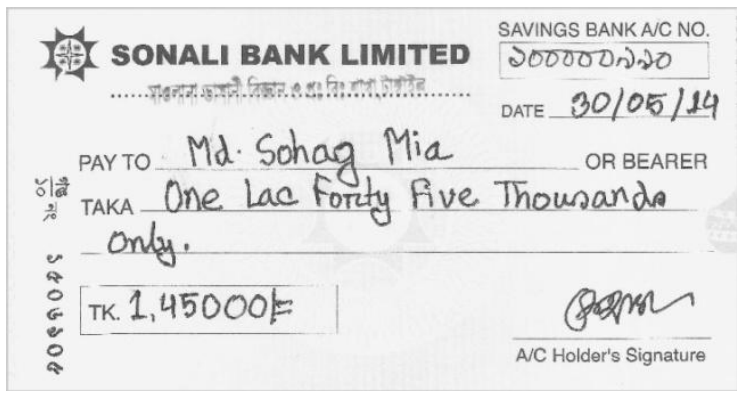

Fig 5: Gray image

\subsubsection{Filtering}

A 3-by-3 median filter is used to blur the background. The characters are slightly blurred, but the background areas are more blurred in the image as in Figure 6.

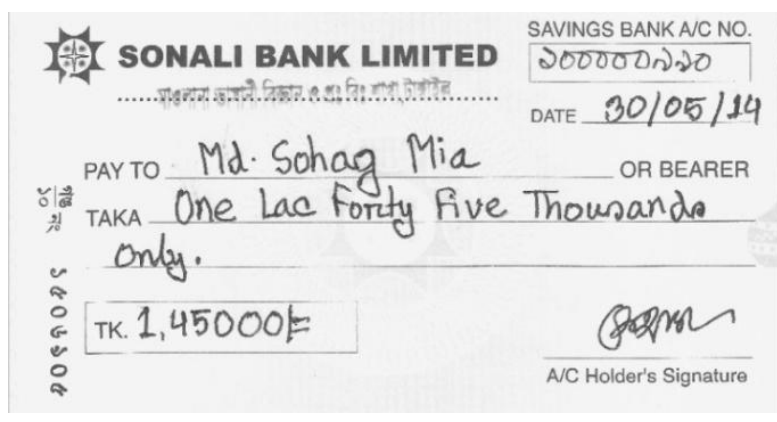

Fig 6: Filtered image

\subsection{Extraction of Courtesy Amount and Signature}

After filtering the image, both courtesy amount and signature is selected by manually from filtered image or set the rectangular area in predefined function to auto select the courtesy amount and signature. In Bangladesh, the courtesy amount is always located at the lower left corner and delimited by a rectangle box in the check. And the signature is always located at the lower right corner in the check. Since 
the size and the aspect ratio of this rectangle are fairly constant for a particular bank, the amount written in the box can be accurately determined and signature written above the line of signature block. Figure 7 shows the cropped courtesy amount and signature from the Cheque.

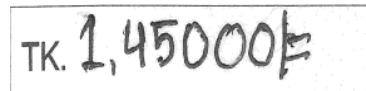

(a)

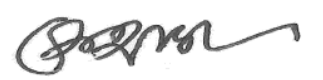

(b)

Fig 7: Cropped image (a) courtesy amount (b) signature

\subsection{Binarization of Courtesy Amount and Signature}

The courtesy amount and signature are converted from Gray scale to binary image that is an image with pixels 0 's (white) and 1's (black). This conversion can take place because it conveys proper information of Numeral Structure. Figure 8 shows a binary image of the courtesy amount and signature, where the ones are drawn in white and zeros are shown in black.

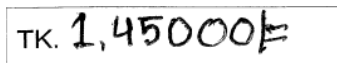

(a)

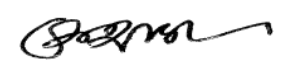

(b)

Fig 8: Binary image (a) courtesy amount (b) signature

\subsubsection{Remove unwanted portion of the cropped image}

Converting into binary image, we have to remove the unnecessary pixels (0) from original image. This is done because we need to develop size independent algorithm.

\section{Algorithm:}

i) Start from top-left corner; repeat for each column and row.

If black pixels is encounter in row/column

Then save column/row

ii) Else don't save column/row.

\subsection{Segmentation Procedure}

The challenge of a segmentation technique lies in the decision of the best cut path to localize an entity to be recognized as a correct isolated character by the recognition system. The literature usually shows three different strategies to perform the segmentation: Segmentation-Recognition, Segmentationbased Recognition and Segmentation Free systems.

Our works are based on the segmentation-based recognition strategy. The aim of this article is to show how we define a new segmentation algorithm taking into account to complementary sets of structural features.

$$
145000=\rightarrow 145000
$$

Fig 9: Segmented digits

\subsection{Feature Extraction}

To extract the feature of the segmented digit and signature we use a rotation and size independent feature extraction method and obtain 26 features for each digit and each signature [15].

\section{Center of the image}

Center of the image can obtain by using following equation:

$$
\begin{aligned}
& \text { Center_x }=\text { width } / 2 \\
& \text { Center_y }=\text { height } / 2
\end{aligned}
$$

Feature1

The first feature is the relation between the height and the width of the image

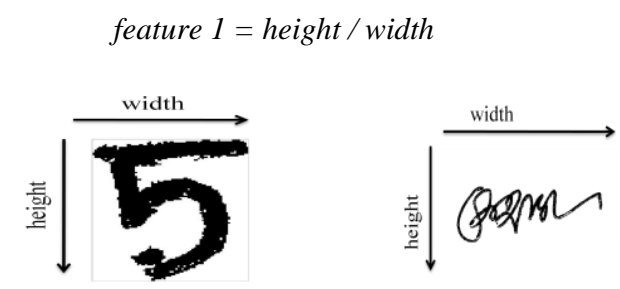

Fig 10: Calculating height $\&$ width of the image

\section{Feature 2 - 26}

These features check how the black pixels are distributed in the image. First the number of pixels inside the image is calculated that is total_pixels of images.

Total_pixel $=$ height $\times$ weight

The feature 2 and 3 are the percentage of black pixels located in the upper and lower areas of images, in other words, the pixels located up and down the central point.

feature $2=$ up_pixels/total_pixels

feature $3=$ down_pixels/total_pixels

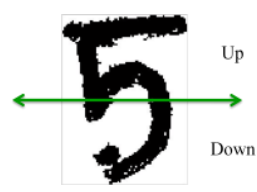

Up

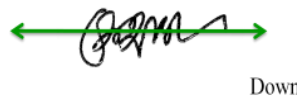

Fig 11: Dividing the image into upper and lower area.

The feature 4 and 5 are the percentage of black pixels located in the left and right areas of images, in other words, the pixels located in the left and right of the central point. feature $4=$ left_pixels/ total_pixels feature $5=$ right_pixels/total_pixels
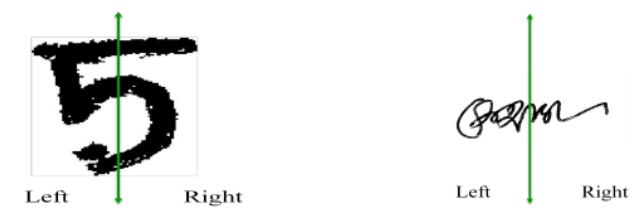

Fig 12: Dividing images into left and right areas.

Now split the image into 4 sub regions and calculate the percentage of black pixels located in every region. Again sub divides every region into four and calculates the percentage of black pixels of those regions. The features 6 to 9 are the percentage of black pixels located in the every sub areas of images. 
feature $_{n}=$ sub_area_pixels ${ }_{n} /$ total_pixels Where $n=6$ to 26
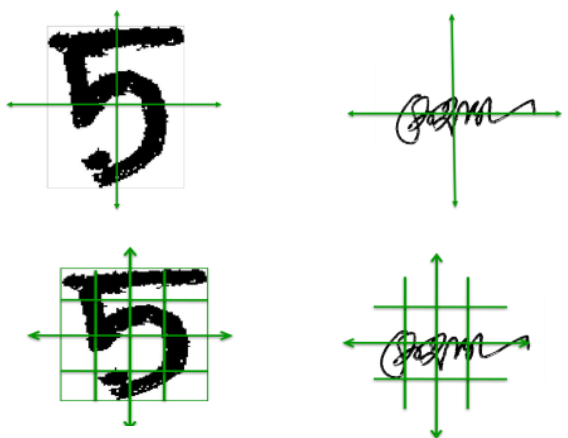

Fig 13: Split the image region

\subsection{Neural network Recognition}

Neural network is composed of a number of nodes called neurons connected by links. Each link has a numeric weight. The neurons are the basic building blocks of neural network $[3,5]$.

\subsubsection{Neural Network Design}

A neural network is employed for courtesy amount and signature recognition. A multilayer feed forward neural network with supervised learning method is more reliable and efficient for this purpose. The network applies back propagation-learning algorithm that is a systematic method for training multiple layer ANNs.

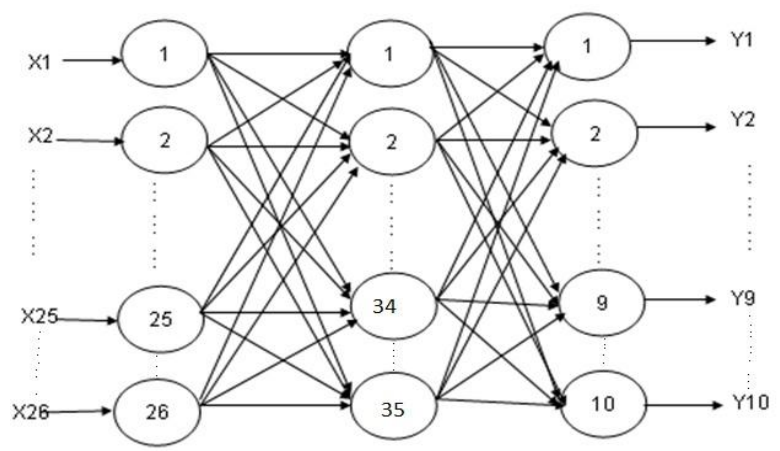

Fig 14: Network design for the system

\subsubsection{Parameters settings}

A Neural Network has several parameters i.e. learning Rate Parameter $(\eta)$, Weights $(w)$, and Momentum $(\alpha)$. A learning rate is design to determine how much the link weights and bias can be modified based on the direction and change rate. Its value should be 0 to 1 . For a given neuron the learning rate can be chosen inversely proportional to the square root of the number of synaptic connection to the neuron.

The weights of the network to be adjusted by using backpropagation algorithm must be initialized by some non-zero values. Initialize weights are randomly chosen between $[-0.5$, $+0.5]$ or $[-0.1,+0.1]$. The weight adjustment is performed using the following equation.

$\Delta w(n)=\eta \cdot \delta(n) \cdot y(n)+\alpha \Delta w(n-1)$

Momentum $(\alpha)$ range 0 to 1 but in 0.9 , found to be most suitable for most application.
Table 1. Initialize value of Network parameters.

\begin{tabular}{|l|c|}
\hline Parameter & Value \\
\hline Hidden Layer & 35 \\
\hline Output Layer & 10 \\
\hline Learning Rate & 0.0001 \\
\hline Total number of epochs & 500 \\
\hline Performance Goal & 0.00000001 \\
\hline Momentum & 0.9 \\
\hline
\end{tabular}

\section{EXPERIMENTAL RESULTS AND PERFORMANCE}

Experimental result has been tested and also measured the performance or accuracy of the system. The proposed system has been implemented using MATLAB which is a high performance language for computing. MATLAB typically used in math and computation, algorithm development, modeling, simulation and prototyping, Data analysis, exploration, Scientific and engineering graphics and application development. The system was trained and evaluated with different forms of handwritten digits and signature provided by both male and female participants. So the overall performance of the system directly depends on the performance of the individual phases. The algorithms and techniques used to read the courtesy amount can be applied for the recognition of the legal amount as well as the signature within a Bank checks. The experiment results are summarized below:

\subsubsection{Training and Recognition:}

Experiment has been done to observe three things: the behavior of the neural network, signature recognition and the digit recognition accuracy. After extraction and segmentation of scanned image, each digit converted into 20 x15 binary images to be analyzed by the neural network. All the samples were split into two groups: the training set and the test set. The training set was comprised of $80 \%$ of the total genuine samples and rests are used for testing the system.

The recognition method has been tested with 50 types of handwritten digit. And every digit has fifty samples. To test signature we collect signature from different persons. We use ten samples of signature to test for an individual. If the train and test set is same the recognition rate is $100 \%$, otherwise the results are above $93 \%$ for digit. For signature recognition the accuracy rate is above $96 \%$ and error rate is only $4 \%$. The overall recognition rates of test data are given bellow.

Table 2. Overall recognition for handwritten digits

\begin{tabular}{|c|c|c|c|}
\hline $\begin{array}{c}\text { Types } \\
\text { of } \\
\text { Digit }\end{array}$ & $\begin{array}{c}\text { No. of } \\
\text { Sample }\end{array}$ & $\begin{array}{c}\text { Correct } \\
\text { recognition } \\
\text { rate (\%) }\end{array}$ & $\begin{array}{c}\text { Error } \\
\text { rate } \\
(\%)\end{array}$ \\
\hline 0 & 50 & 95 & 5 \\
\hline 7 & 50 & 95 & 5 \\
\hline 2 & 50 & 95 & 5 \\
\hline 3 & 50 & 90 & 10 \\
\hline 2 & 50 & 89 & 11 \\
\hline 5 & 50 & 95 & 5 \\
\hline 5 & 50 & 95 & 5 \\
\hline
\end{tabular}




\begin{tabular}{|c|c|c|c|}
\hline & 50 & 90 & 10 \\
\hline & 50 & 95 & 5 \\
\hline & 50 & 95 & 5 \\
\hline Total & $\mathbf{5 0 0}$ & $\mathbf{9 3 . 4}$ & $\mathbf{6 . 6}$ \\
\hline
\end{tabular}

Table 3. Overall recognition rate for Signature

\begin{tabular}{|c|c|c|c|}
\hline $\begin{array}{c}\text { Type of } \\
\text { Signature }\end{array}$ & $\begin{array}{c}\text { No. of } \\
\text { Sample }\end{array}$ & $\begin{array}{c}\text { Correct } \\
\text { recognitio } \\
\text { n rate } \\
(\%)\end{array}$ & $\begin{array}{c}\text { Error rate } \\
(\%)\end{array}$ \\
\hline pabag & 10 & 94 & 6 \\
\hline Bme & 10 & 97 & 3 \\
\hline glom. & 10 & 95 & 5 \\
\hline sutarmun & 10 & 96 & 4 \\
\hline Soma & 10 & 98 & 2 \\
\hline Itobat & 10 & 96 & 4 \\
\hline Strons & 10 & 95 & 5 \\
\hline cutbut & 10 & 98 & 2 \\
\hline S & 10 & 95 & 5 \\
\hline Total & 90 & 96 & 4 \\
\hline
\end{tabular}

And finally the figure 15 shows the training performance of the system.

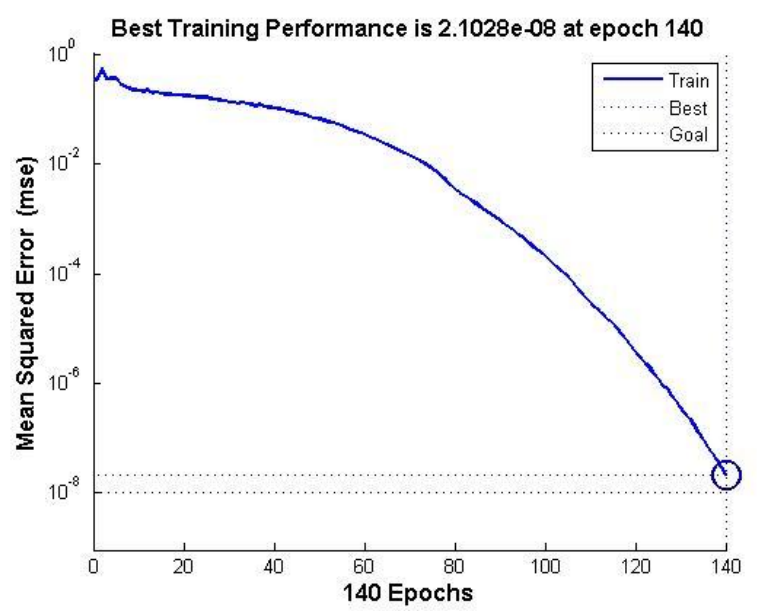

Fig 15: performance of the system

\section{CONCLUSION}

The proposed system has developed a method for Recognition handwritten numerical characters and signature. The system is tested by 50 items for each type of numerical characters and 10 items for signature and obtains the result where overall success rate of the system is $93.4 \%$ for courtesy amount and for signature the accuracy rate is $96 \%$ which meet the expectation of the thesis. So the system achieves its desired expectation. In future, we extend the proposed system to increase the accuracy and HMM will used to detect the hand written courtesy amount and signature.

\section{REFERENCES}

[1] Agarwal A., Gupta A., and Hussein K., "Bank Check Analysis and Recognition by Computers" in Handbook of Character Recognition and Document Image
Analysis. Editors: H.Bunke, and P.S.P. Wang. World Scientific, 1997.

[2] Asaduzzaman A., Parveen M. S., and Ali M. G., "Detection of Bangla Numbers Using Artificial Neural Network", 5th ICCIT 2003, Jahangirnagar University, 19-21 December 2003, pp. 347-350

[3] Beale R., and Jackson T., Neural Computing: An Introduction, IOP Publishing Ltd. 1990.

[4] Blumenstein M., and Verma S., "A Neural Based Segmentation and Recognition Technique for Handwritten Words". IEEE International Conference on Neural Networks, Vol. 3, pp.1738-1742, 1998

[5] Bose N. K., and Liang P., "Neural Network Fundamentals with Graphs, Algorithms, and Applications", International edition, 1996

[6] Casey R. G., and Lecolinet E., "A Survey of Methods and Strategies in Character Segmentation", in IEEE Transactions on Pattern Analysis and Machine Intelligence, Vol. 18(7), pp. 690-706, 1996.

[7] Dewangan S. K., "Real Time Recognition of Handwritten Signatures without Segmentation Using Artificial Neural Network" MECS (http://www.mecspress.org/), Vol. 5 pp. 30-37, April 2013

[8] Dey S., "Adding Feedback to Improve Segmentation and Recognition of Handwritten Numerals", Master Thesis, Massachusetts Institute of Technology. 1999.

[9] Guillevic D., and Suen C. Y., "Recognition of legal amounts on bank Cheques", Pattern Anal. Appl. Vol. 1 (1), pp. 28-41, 1998.

[10] Haykin S., "Neural Networks: A Comprehensive Foundation", Second edition, Pearson Education Asia, 2001

[11] Kaufmann G., and Bunke H., "A system for the automated reading of check amounts - Some key ideas", Proceedings of the 3rd International Association of Pattern Recognition Workshop on Document Analysis Systems. Nagano, Japan, pp. 302315, 1998.

[12] Lethelier E., Leroux M., and Gilloux M., "An Automatic Reading System for Handwritten Numeral Amounts on French checks." 3rd International Conference on Document Analysis and Recognition, vol 1:92-97, 1995.

[13] Marinai S., Marino E., and Soda G., "Font adaptive word indexing of modern printed documents", IEEE Trans. on Pattern Anal. and Machine Intell, Vol. 28(8) pp. 1187-1199, 2006.

[14] Mashiyat A.S., Mehadi A.S., Talukder K.H., "Bangla offline Handwritten Character Recognition Using Superimposed Matrices", 7th International Conference on Computer and Information Technology, pp. 610614, December 2004.

[15] Miah B. A., Haque S. M. A., Mazumder R., Rahman Z., "A New Approach for Recognition of Holistic Bangla Word using Neural Network," IFRSA International Journal of Data Warehousing \& Mining, Vol. 1, issue. 2, pp. 139-141, Nov 2011. 
[16] Mollah M. K. I., and Talukder K. H., "Bangla Number Extraction and Recognition from Document Image", 5th ICCIT 2002, East West University, pp. 200-206, 27-28 December 2002.

[17] Pal U., Belad A., and Choisy C., "Touching numeral segmentation next term using previous term water next term reservoir concept", Pattern Recognition Letters, Vol. 24(1-3), pp. 261-272, 2003.

[18] Palacios R., Gupta A., and Wang P. S., "Feedback based architecture for reading courtesy amounts on checks," Journal of Electronic Imaging, Vol.12 (1), pp. 194-202, 2003.

[19] Punnoose J., "An Improved Segmentation Module for Identification of Handwritten Numerals", Master Thesis, Massachusetts Institute of Technology. 1999.
[20] Shah M. S., Haque S. M. A., Islam M. R., Ali M. A., and Hasan M. S., "Automatic Recognition of Handwritten Bangla Courtesy Amount on Bank Checks" IJCSNS International Journal of Computer Science and Network Security, VOL.10 No.12, December 2010

[21] Suen C., Lam L., Guillevic D., Strathy N., Cheriet M., Said J., and Fan R., "Bank check processing system", Int. J. Imag. Syst. Technol. Vol. 7, pp. 392-403, 1996.

[22] Talele A. K., Nalbalwar S. L., and Rane M. E., "Automatic Recognition and Verification of Handwritten Legal and Courtesy Amounts in English Language Present on Bank Cheques" International Journal of Computer Applications (IJCA)(0975-8887) Volume 21- No.8, May 2011. 